\section{GLOMERULAR DISORDERS}

\section{9 \\ MESANGIAL IgA NEPHROPATHY IN HLA-IDENTICAL SIBLINGS} WITH SIMILAR BLOOD TYPES

Few reports have been made on intrafamilial cases of IgA nephropathy. Recently, a study was carrled out on two siblings with the simflar HLA and blood types. Patlent 1 (brother) developed gross hematuria and protelnuria at age 7 . Patient 2 (slster) developed microscopic hematuria at the same age.

Neither patient exh1b1ted nerve deafness nor occular defects; renal function was also normal. Microscoplc hematuria was observed in three other siblings of the father.

Renal biopsles were performed and subsequent electron mlcroscoplc observation revealed evidence of widespread paramesanglal electron-dense deposits.

Immunofluorescence showed these deposits to be IgA. IgG, $\mathrm{C}_{3}$ and Fibrin-Fibrinogen were also detected in glomeruli in both patients.

Serotyping revealed Identical HLA, A and B locus, which were HLA-Al1, BW48/A11, BW48, respectively.

Blood type was also identical except for Lew1s. ABO, Rh, MNS, and $P$ were $A_{1}, C c D E e, M s N s, P_{2}$, respectively, in both patients.

These findings are of paticular interest in view of the assoclation of IgA nephropathy with genetically controlled, immune response. \section{CLINICOPATHOLOGICAL CORRELATIONS IN FAMILIAL}

\author{
The children Hoshikawa, N., White, R.H.R. \& Cameron, A.H...
}

The findings are reported in 38 patients with FH. Those with neurosensory deafness, heavy proteinuria or chronic renal failure in the patient and/or family generally had a progressive clinical course. Their biopsies showed segmental glomerular sclerosis usually with interstitial foam cells; on electron microscopy (EM) there was usually fibrillary replication of the lamina densa with a 'basket weave' pattern. These adverse features were more pronounced when there was deafness in the patient or family, although families without deafness are considered to fall within the spectrum of Alport's syndrome. In contrast, patients from families showing neither deafness, heavy proteinuria nor chronic renal failure ran a non-progressive course (FH). Their biopsies showed little or no glomerular changes other than attenuation of the lamina densa on EM.

Deafness, heavy proteinuria, segmental sclerosis and foam cells were not often present before the age of 10 years in children with Alport's syndrome, whereas the 'basket weave' pattern of the lamina densa was found in all three children biopsied under 5 years old. We therefore enphasize the importance of EM in the differential diagnosis from benign FH.

111 CLINICAL PATHOLOGICAL CORRELATIONS IN RECURRENT AND PERSISTENT HEMATURIA SYNDROMES (RPHS). A report from the International Study of Kidney Disease in Children.

We report on 75 children satisfying the following criteria: (1) hematuria: 3 or more $\mathrm{RBCs} / \mathrm{mm}^{3}$ or trace or more hemastix; (2) recurrent: 2 or more episodes within six months - negative intervening specimens; (3) persistent: consistent positives over 6 months; (4) no family history of nephritis. The following immunological sub-groups were identified: I: Mesangial IgA (12): II: Mesangial IgM (10); III: Vascular C3 (27); IV: Peripheral (epimembranous) IgG (3); V: Mesanglal IgG (2); VI: Essentially negative (21). Elevated $\mathrm{Cr}$ (>2SD) was found in I $1 / 12$; II $0 / 10$; III $4 / 27$; IV $0 / 3 ; V 0 / 2 ; V I 4 / 21$. Elevated diastolic BP was noted in I: $1 / 12$ and elevated systolic $B P$ in $I: 2 / 12$ and III: $1 / 27$. No significant difference in the pattern of hematuria was noted with respect to gross vs microscopic or intermittent vs persistent. Three patients in group IV had typical epimembranous deposits with microscopic hematuria, only $1 / 3$ had proteinuria. Mesangial hypercellularity was seen in all groups. Focal global or segmental glomerulosclerosis was seen in I 10/12; II $3 / 10$; III $12 / 27$; IV $1 / 3 ; \mathrm{V} 1 / 2 ;$ VI $7 / 21$. Tubular atrophy was seen in I $9 / 12$; II $2 / 10$; III $8 / 27$; IV $0 / 3$; V 0/2; VI $5 / 21$. Serum Ig $s$ revealed only increased mean IgA levels in group 1 .

RPHS are associated with several immunopathological patterns. These include mesangial Ig deposits (I,II,IV), vascular C3 (III). epimembranous IgG (IV) and negative (VI). Elevated serum creatinine, glomerulosclerosis, or tubular atrophy was more commonly seen in groups I, III and VI.
112 BERGERS DISEASE - HENOCH SCHONLEIN NEPHRITIS WITHOUT THE RASH Meadow S R, Scott D G, St James's University Hospital, Leeds, England.

There are similarities in the clinical and renal manifestations of Bergers disease (mesangial IgA disease) and HenochSchonlein nephritis. The diagnosis of Henoch-Schonlein syndrome is made only when the purpuric rash is seen. It can be suggested that some cases of Bergers disease are Henoch-Schonlein syndrome without the rash. The following family illness suggests that this is so.

Identical 7 yr old twin boys each had a proven adenovimus infection at the same time. A few days later one developed florid Henoch-Schonlein purpura, severe alimentary symptoms and transient joint symptoms. He had an acute nephritic syndrome which progressed to nephrotic symdrome and renal insufficiency. Biopsy showed severe proliferative glomemulonephritis with crescents and marked deposition of IgA, IgG, $C_{3}$ and fibrin. The twin presented with haematuria and abdominal pain but no rash. The bouts of haematuria recurred but gradually subsided to merely microscopic haematuria. His biopsy showed mesangial proliferative glomerulonephritis with mesangial deposits of IgA and to a less extent $I_{8 G}$ and $C_{3}$. The appearance was characteristic of Bergers disease.

Immunological studies have not revealed why these two identical twing responded to the same provocation in different ways. Their illness suggests that Bergers disease may be considered a variety of Henoch-Schonlein nephritis (without the rash).

\section{PATHOLOGICAL CHARACTERISTICS \\ 113 PATHOLOGICAL CHARACTERISTICS \\ Nakahara, C. Aosai, F., Hasegawa, O. Ito, H., Matsuo, N., Hajikano, H. \\ Sakaguchi, H.Dts. of Pediatrics \& Pathology, Tokyo Metropolitan Children's Hospital, Tokyo, Japan.}

The present study was carried out to determine clinical and immunopathological features of IgA nephropathy in children.

Of 224 children who underwent renal biopsy between $1975 \& 1979$ and on whom the specimens were studied by immunoffuorescence, 35 cases (male 22, female 13, ages 4 to 17 years) of IgA nephropathy were detected. Their clinical, immunological and histological characteristics were compared to that of 71 cases of IgA nephropathy found in 311 biopsied specimens of adult patients. All the specimens were analysed by a routine light microscopy and immunofluorescent study for immunoglobulins, $\beta 1 C, \beta 1 E$, properdin and fibrinogen. In order to estimate mesangial cell proliferation, matricial increase, mesangial deposition, and tubulo-interstitial changes quantitatively, each specimen was analysed on our scoring system. Results are summerized as follows.

1) Clinical manifestations: $23(65.7 \%)$ were presented by chance proteinuria and/or hematuria, and $7(20 \%)$ by macroscopic hematuria. During the course of illness, $5(14.3 \%$ ) developed nephrotic syndrome and $15(42.5 \%)$ macroscopic hematuria. They were followed over a mean period of 52.1 months: 9 improved, 28 unchanged, and 1 deteriorated. It may sugges better prognosis in pediatric patients despite a higher incidence of heavy proteinuria and

2) Serum IgA levels: Serum IgA levels were elevated in 7 of 26 children above 10 years of age but remained within normal range in all of 9 children below 10 years. 3) Immunopathological study: In comparison with adult cases, mesangial cell proliferation was more prominent in majority of the 35 cases, but increase in mesangial matrix and deposits, and tubulo-interstitial changes were milder. Mesangial immunofluor escence was unequivocally demonstrated in all of the 35 children, being composed of $\operatorname{IgA} \bar{s} \beta 1 C$ in $4, \lg A \bar{c} \beta 1 C$ in 12 , and complex immunogloblins $\bar{c} \beta 1 C$ in 19 . In the adult patients, however, $\beta 1 C$ was stainable in the mesangium in all but one of the 71 cases.

The present data suggests that IgA nephropathy in children differs distinctively from that of adults in clinical, immunological, and pathological manifestations.
114

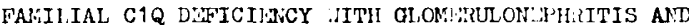

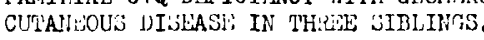
Eci ja,J.L; Wanchez-Ba, le,M; Mampaso, $\mathrm{ir}$; Zambrano,A.and Leyva, F. Hospital Niño Jesús and C. Lamon y Cajal. Madrid. Spain

A C1q deficiency with outaneous and renal disease was found in three siblings. Two brothers and a sister aced 12, 11 and? years old, with clinical features resembline those of RothmundThomson Syrdrome were studied because of episodes of cross hematuria in two patients and microsropic in the other.

lenal furction and cryoglobulins wore normal, scrum IrM was nlevated and Im was positive in all three. N:A wnre only detected in two patients. Serologic studies showed a total lack of CH5O hemolytic activity, undetectable Clq and restoration of the defect when purified human CIq was added to the assa\%. Yesanrial Proliferative Glomerulon phritis with diff:se lomerular denosits of $I \sim l$ and $C 3$ vas scen by renal biopsy and cutareous hystological studies were those of iothmund-I'horison Sirndrome in all three patierts. Ho clinical and seroloric alnormalities were found in the mother and in the remainder three other sillines. Th $c$ father presented AllA at a low tittle and a serum C1n decreasnd to a $50 \%$ of its normal value. 106 members of the farily tested a rainst any cutaneous or reval clinical symptoms were normal.

A familinl incidence of three not nreviously reported combined discases hes bee: found. Cerolo;ic dat: and tile renal affectation hichly sur, est a sLE-like conrective tissue discrse, in which the conserital lack of $\mathrm{Cla}$ scerrs to ic the predowinant factor. 